\title{
The Effect of Flux Flow on Self-Field Instability
}

\author{
Lawrence Dresner
}

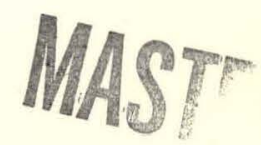




\section{DISCLAIMER}

This report was prepared as an account of work sponsored by an agency of the United States Government. Neither the United States Government nor any agency Thereof, nor any of their employees, makes any warranty, express or implied, or assumes any legal liability or responsibility for the accuracy, completeness, or usefulness of any information, apparatus, product, or process disclosed, or represents that its use would not infringe privately owned rights. Reference herein to any specific commercial product, process, or service by trade name, trademark, manufacturer, or otherwise does not necessarily constitute or imply its endorsement, recommendation, or favoring by the United States Government or any agency thereof. The views and opinions of authors expressed herein do not necessarily state or reflect those of the United States Government or any agency thereof. 


\section{DISCLAIMER}

Portions of this document may be illegible in electronic image products. Images are produced from the best available original document. 


\section{Printed in the United States of America. Available from National Technical Information Service \\ U.S. Department of Commerce \\ 5285 Port Royal Road, Springfield, Virginia 22161 \\ Price: Printed Copy $\$ 4.00$; Microfiche $\$ 3.00$}

This report was prepared as an account of work sponsored by the United States Government. Neither the United States nor the Energy Research and Development Administration/United States Nuclear Regulatory Commission, nor any of their employees, nor any of their contractors, subcontractors, or their employees, makes any warranty, express or implied, or assumes any legal liability or responsibility for the accuracy, completeness or usefulness of any information, apparatus, product or process disclosed, or represents that its use would not infringe privately owned rights. 
ORNL/TM-5986

Contract No. W-7405-eng-26

FUSION ENERGY DIVISION

THE EFFECT OF FLUX FLOW ON SELF-FIELD INSTABILITY

Lawrence Dresner

Date Published - August 1977

NOTICE This document contains information of a preliminary nature.

It is subject to revision or correction and therefore does not represent a final report.

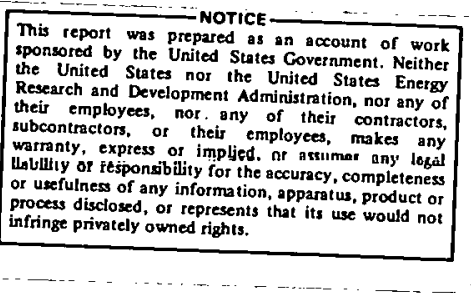

Prepared by the

OAK RIDGE NATIONAL LABORATORY

Oak Ridge, Tennessee 37830

operated by

UNION CARBIDE CORPORATION

for the

ENERGY RESEARCH AND DEVELOPMENT ADMINISTRATION 


\section{THIS PAGE \\ WAS INTENTIONALLY \\ LEFT BLANK}




\section{CONTENTS}

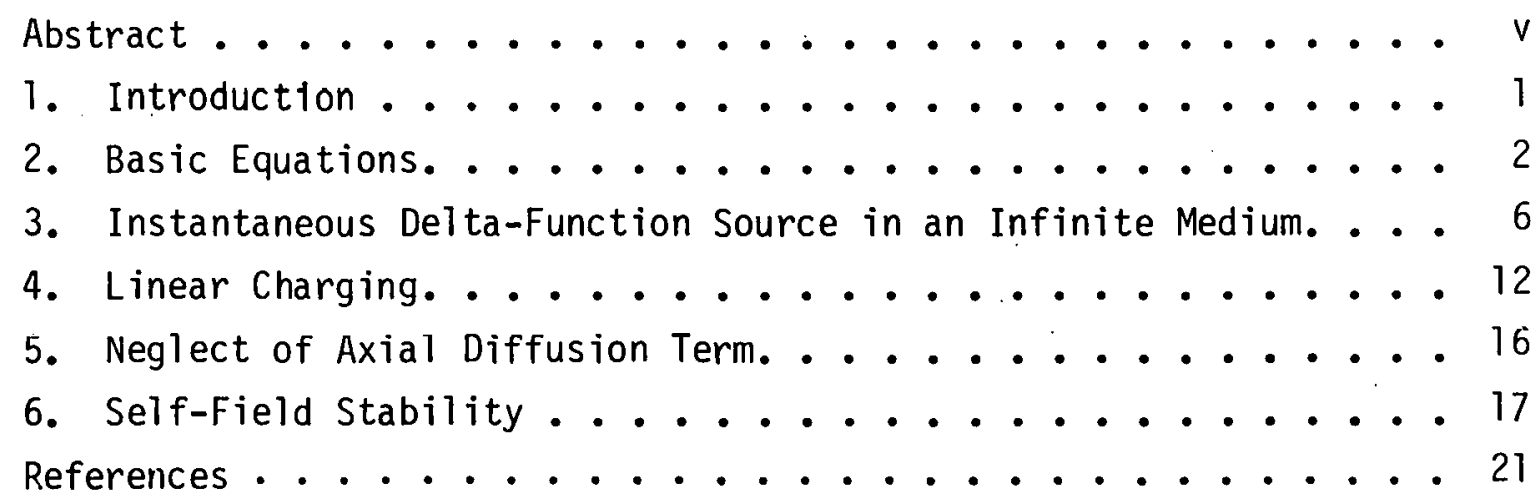


THIS PAGE

\section{WAS INTENTIONALLY LEFT BLANK}




\section{ABSTRACT}

Flux flow causes type II superconductors to develop resistance continuously rather than suddenly as transport current increases. This means that the distribution of current among the filaments in a composite conductor is determined not only by their inductive coupling but also by the longitudinal resistance they develop as they begin to carry current. The current distribution is calculated in two cases, taking flux flow into account: a composite clamped suddenly across a constant-current source and a composite charged with current at a uniform rate. The results of the latter problem are used to show that slowly charged conductors will be much more stable against self-field instability than is indicated by purely inductive calculations. 


\section{INTRODUCTION}

In a recent paper, ${ }^{1}$ Wilson noted that Duchateau and Turck's theory ${ }^{2}$ of self-field instability seemed pessimistic when compared with measurement. In Wilson's view, the distribution of current density used by Duchateau and Turck is wrong because it fails to account for the progressive decay towards uniformity caused by flux flow. Duchateau and Turck calculate the current distribution in a conductor on the basis of purely inductive coupling between perfectly conducting strands, with the restriction that the current in a filament never exceeds its critical current. This is the same as the assumption that the resistivity of the superconductor jumps suddenly from zero to its full normal value at the critical current density.

In reality, flux flow causes type II superconductors to develop resistance continuously rather than suddenly as the transport current increases. This means that the distribution of the current among the filaments is determined not only by their inductive coupling but also by the longitudinal resistance they develop as they begin to carry current.

In the simplest case of an untwisted composite, the purely inductive calculation of Duchateau and Turck leads to a current distribution in which a sheath of outer filaments is saturated and the inner filaments carry no current at all. Wilson has estimated the time it would take this current distribution to decay, and he finds it to be short compared with the times generally anticipated for charging toroidal field coils. Consequently, the current distribution of Duchateau and Turck will never exist at all in a slowly charged magnet. This is the reason for Duchateau and Turck's overly pessimistic expectations, as set forth by Wilson.

The purpose of the present paper is twofold: first, to improve Wilson's simple estimates of the decay time, and second, to calculate the distribution of current among the filaments of a conductor being charged at a uniform rate. 


\section{BASIC EQUATIONS}

Let us consider a composite conductor in the form of an infinite slab, the faces of which are perpendicular to the $y$-axis (see Fig. 1).

QRNL /DWG/FED-7734

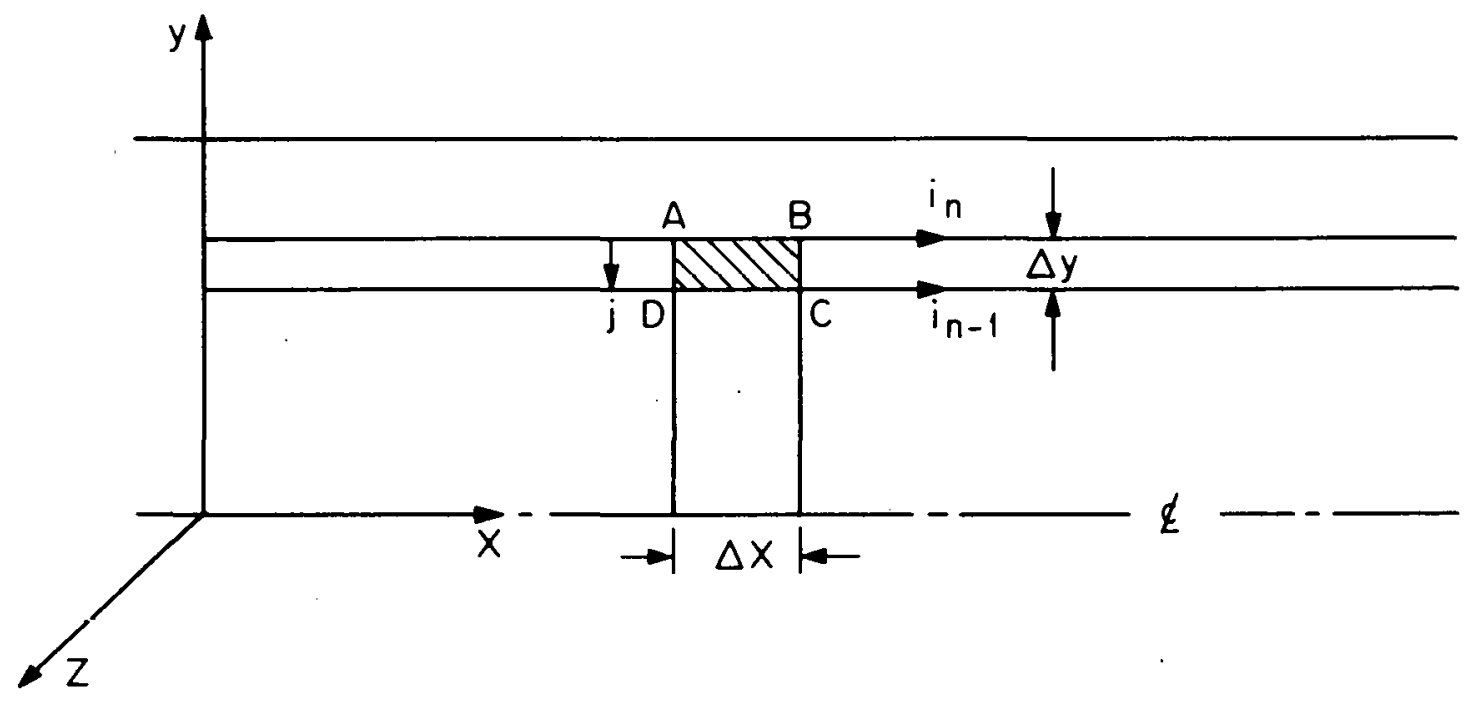

Fig. 1. Sketch of the slab conductor showing the coordinate system.

Let the midplane of the slab be the $(x, z)$ plane. The superconductor is distributed in thin sheets parallel to the faces and is equally spaced at intervals $\Delta y$. The $n^{\text {th }}$ sheet carries a current $i_{n}$ in each unit of its length along the z-axis; the current flows in the $x$ direction. Above the midplane, between sheets $n-1$ and $n$, the self-field $B$ of the sheet currents points in the $z$ direction and is given in magnitude by $B=\mu_{0} \sum_{k=1}^{n-1} i_{k}$.

As the currents and field change with time, the rate of change of flux through the shaded rectangle $A B C D$ is

$\Delta x \Delta y \frac{\partial B}{\partial t}=\mu_{0} \Delta x \Delta y \frac{\partial}{\partial t} \sum_{k=1}^{n-1} i_{k}$ 
The right-hand side of Eq. (2) gives the voltage around the path $A B C D$. Along the leg $A B$; the voltage drop is $i_{n}\left(\rho_{n} \Delta x / n \Delta y\right)$, where $\rho_{n}$ is the fluxflow resistivity in the $n^{\text {th }}$ sheet and is a function of the current $i_{n}$, and $n$ is the volume fraction of superconductor. The voltage drop along the leg $B C$ is $j(x+\Delta x) \rho_{c u} \Delta y$, where $j$ is the current density flowing in the copper perpendicular to the superconducting sheets and towards the center line. Thus,

$$
\begin{gathered}
\mu_{0} \Delta x \Delta y \frac{\partial}{\partial t} \sum_{k=1}^{n-1} i_{k}=i_{n}\left(\frac{\rho_{n} \Delta x}{n \Delta y}\right)-i_{n-1}\left(\frac{\rho_{n-1} \Delta x}{n \Delta y}\right)+j(x+\Delta x) \rho_{c u} \Delta y \\
-j(x) \rho_{c u} \Delta y .
\end{gathered}
$$

If $\Delta x$ and $\Delta y$ are small, we can write this as

$\mu_{0} \cdot \frac{\partial}{\partial t} \int_{0}^{y} i d y=\frac{1}{\eta} \frac{\partial}{\partial y}(i \rho)+\Delta y \rho_{c u} \frac{\partial j}{\partial x}$,

where $i$ is the current in the superconducting sheet at the position $y$, etc. Conservation of current demands that

$\left(\sum_{k=1}^{n-1} i_{k}\right)_{x}+j \Delta x=\left(\sum_{k=1}^{n-1} i_{k}\right)_{x+\Delta x}$

or

$\Delta y j=\frac{\partial}{\partial x} \int_{0}^{y} i d y$.

Then

$\mu_{0} \frac{\partial}{\partial t} \int_{0}^{y} i d y=\frac{1}{n} \frac{\partial}{\partial y}(i \rho)+\rho_{c u} \frac{\partial^{2}}{\partial x^{2}} \int_{0}^{y} i d y$

or, after we differentiate with respect to $y$,

$\mu_{0} \frac{\partial i}{\partial t}=\frac{1}{n} \frac{\partial^{2}}{\partial y^{2}}(i \rho)+\rho_{c u} \frac{\partial^{2} j}{\partial x^{2}}$ 
In an ordinary ohmic medium, $\rho$ may be taken to be constant, but of course we cannot make this assumption for superconductors. For superconductors, it is usually assumed that $\rho$ is a step function of current density, jumping sharply from $\rho=0$ to $\rho=\rho_{s c}$, the full normal resistivity, at the critical current density $J_{C}$ (see the top half of Fig. 2). In strict point of fact, the transition from zero to full normal resistivity takes place rapidly but continuously, as shown schematically in the bottom half of Fig. 2. Here the width of the transition zone has been greatly exaggerated along the J-axis. The lower part of the $\rho-J$ curve can be approximated reasonably well by

$\rho=\rho_{S C}\left(\frac{\mathrm{J}}{J_{0}}\right)^{m-1}$,

where $J_{0}$ is a reference current density and $m$ is a number around 30 or $40 .{ }^{3}$ We use this form henceforth.

If we denote by $i_{0}$ the current in one sheet when the current density has the value $J_{0}$, and set $g=i / i_{0}$ and $\tau=\rho_{s c} t / \eta \mu_{0}$, we get

$\frac{\partial g}{\partial \tau}=\frac{\partial^{2}}{\partial y^{2}}\left(g^{m}\right)+\left(\frac{n \rho_{c u}}{\rho_{S C}}\right) \frac{\partial^{2}-g}{\partial x^{2}}$.

The ratio $\mathrm{g}$ is a dimensionless measure of the current distribution in the slab. The variable $\tau$ is a measure of the time with the dimensions of length squared.

In the problems we consider below, the last term on the right-hand side of Eq. (8) will always be negligible compared with the first term on the right-hand side, except very near the current leads. For this reason, we now turn our attention to the current distribution determined by the equation

$$
\frac{\partial g}{\partial \tau}=\frac{\partial^{2}}{\partial y^{2}}\left(g^{m}\right)
$$



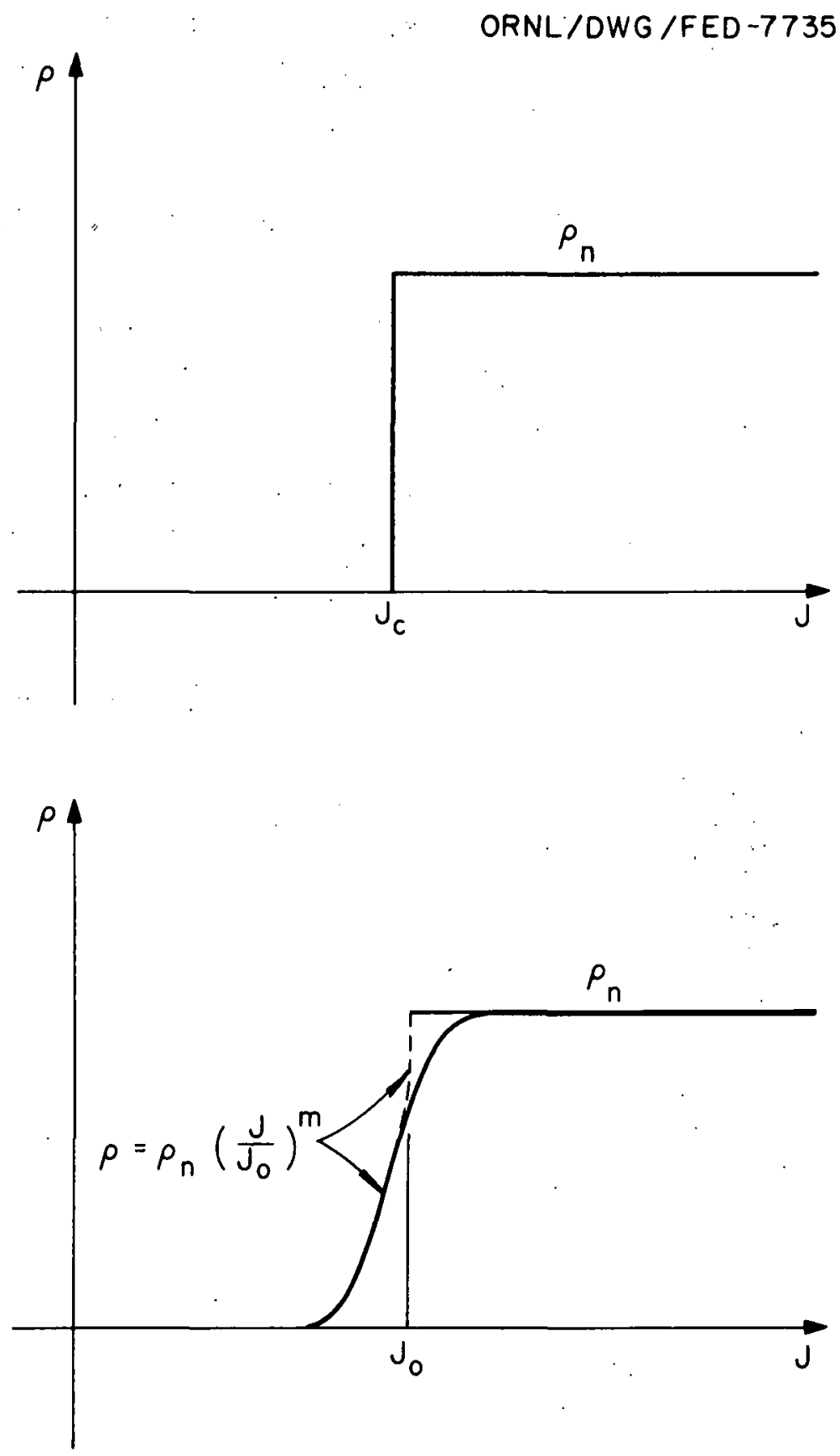

Fig. 2. Resistivity of the superconductor according to the critical-state model (top) and the flux-flow model (bottom). 
After we solve Eq. (9) for the main situation we are interested in below, we verify that the neglect of the last term on the right-hand side of Eq. (8) is justified.

\section{INSTANTANEOUS DELTA-FUNCTION SOURCE IN AN INFINITE MEDIUM}

If $m$ were equal to 1 , Eq. (9) would be the ordinary diffusion equation. The solutions of the ordinary diffusion equation in an infinite medium for an instantaneous delta-function source $\delta(x, t)$ are shown schematically in Fig. 3 for three times, $\tau_{1}<\tau_{2}<\tau_{3}$. Now imagine the slab between $y=U$ and $y=a$ to be half the conductor. The infinite-medium solutions are then good even for the finite slab for times short enough so that at

\section{ORNL / OWG / FED -7736}

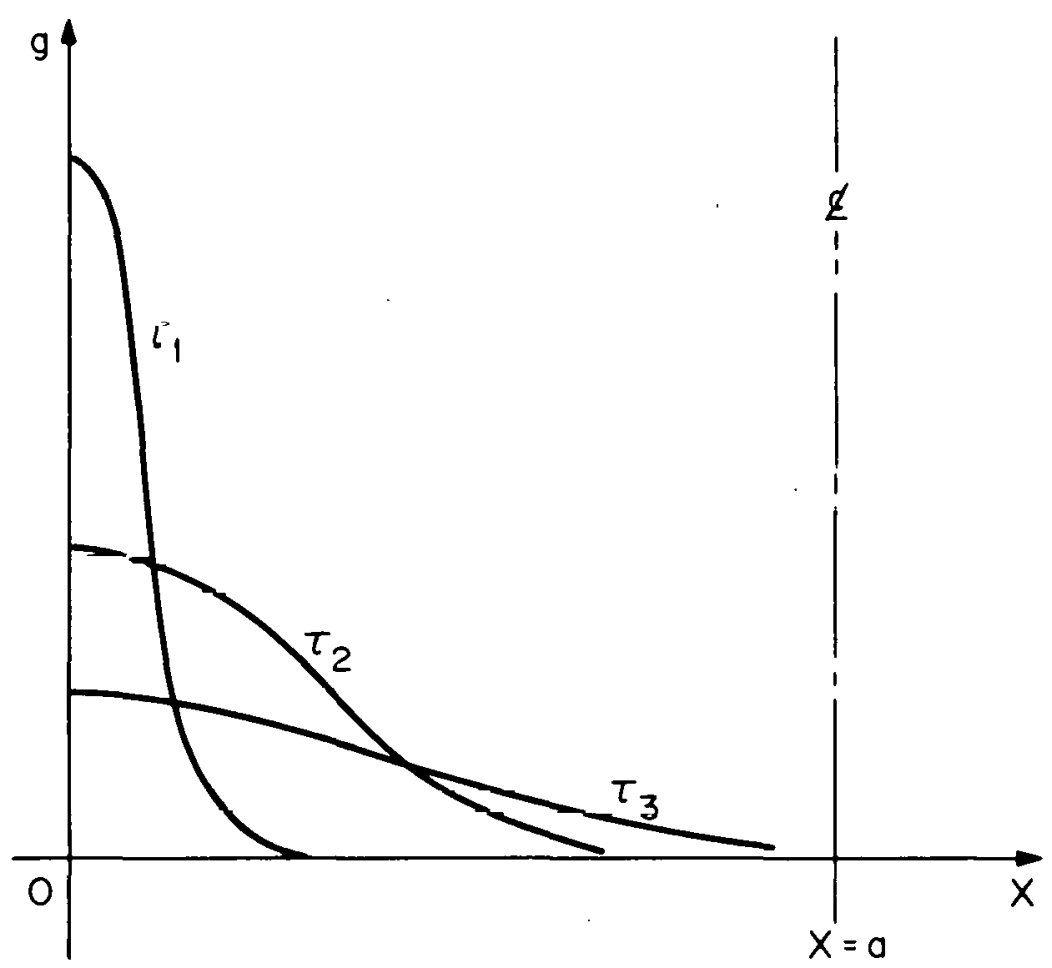

Fig. 3. g-profiles (schematic) for the ordinary diffusion equation with an instantaneous delta-function source. 
$x=a, g$ is very small. The infinite-medium solutions are good at least for times $<\tau_{3}$. A similar conclusion holds when $m>$. 1. But whereas we already know the shape of the g-profiles in Fig. 3 when $m=1$ (Gaussian), we still have to calculate it when $m>1$.

The instantaneous delta-function source $\delta(x, t)$ is of practical interest, for if we suddenly clamp a long superconductor across the ends of a constantcurrent power supply, the current at first lies at the outer surface. Hence we can use the infinite-medium solution to study at least the early stages of current redistribution.

The infinite-medium solution can be found by the method of similarity solutions described by Birkhoff ${ }^{4}$ and by Ames. 5 If we set

$g(y, \tau)=\tau^{-1 /(m+1)} F[y / \tau 1 /(m+1)]$

and substitute into Eq. (9), we get the following ordinary differential equation for the function $F$ of the variable $\xi=y / \tau^{T /(m+1)}$ :

$(m+1) \frac{d^{2}\left(F^{m}\right)}{d \xi^{2}}+\xi \frac{d F}{d \xi}+F=0$

The boundary conditions which $F$ must obey are

$\left(\frac{d F}{d \xi}\right)_{\xi=0}=0 \leftrightarrow\left(\frac{\partial g}{\partial y}\right)_{y=0}=0$

and

$F(\infty)=0 \leftrightarrow g(\infty, \tilde{\tau})=0$

One further condition, which arises from the initial presence of a delta-function source and serves to determine the normalization of $F$, can be obtained as follows. We integrate Eq. (9) over all positive y and, using Eq. (12), find that

$\frac{d}{d \tau} \int_{0}^{\infty} g d y=0$. 
Thus

$$
\int_{0}^{\infty} g d y=\text { constant }=\int_{0}^{\infty} F(\xi) d \xi .
$$

We can integrate Eq. (11) once immediately:

$(m+1) \frac{d\left(F^{m}\right)}{d \xi}+\xi F=0$

The right-hand side of Eq. (15), a constant of integration, must be zero in order to satisfy the boundary condition, Eq. (12a). A second integration gives

$\frac{m(m+1)}{m-1} F^{m-1}+\frac{\xi^{2}}{2}=\frac{\xi_{0}^{2}}{2}$

where $\xi_{0}^{2} / 2$ is a constant of integration. Thus, $F=0$ for $\xi>\xi_{0}$, while for $\xi<\xi_{u}$.

$F(\xi)=\left[\frac{m-1}{2 m(m+1)}\left(\xi_{0}^{2}-\xi^{2}\right)\right]^{1 /(m-1)}$.

The constant $\xi_{0}$ is determined by the normalization, Eq. (14).

Equation (17) gives the profile of $\mathrm{g}$ at any instant of time; its shape is the counterpart of the Gaussian shape for the ordinary diffusion equation. The profile is shown in Fig. 4, where $F(\xi) / F(0)$ is plotted against $y / y_{0}=\xi / \xi_{0}$ for $m=20,30$, and 40 . The shape of the diffusing current density distribution is almost rectangular for large $\mathrm{m}$.

Next, we need to estimate how long it will take for the current distributions diffusing in from each boundary to reach the center line; beyond this time the infinite-medium solutions are no longer valid. From Eqs. (14) and (17) we obtain 


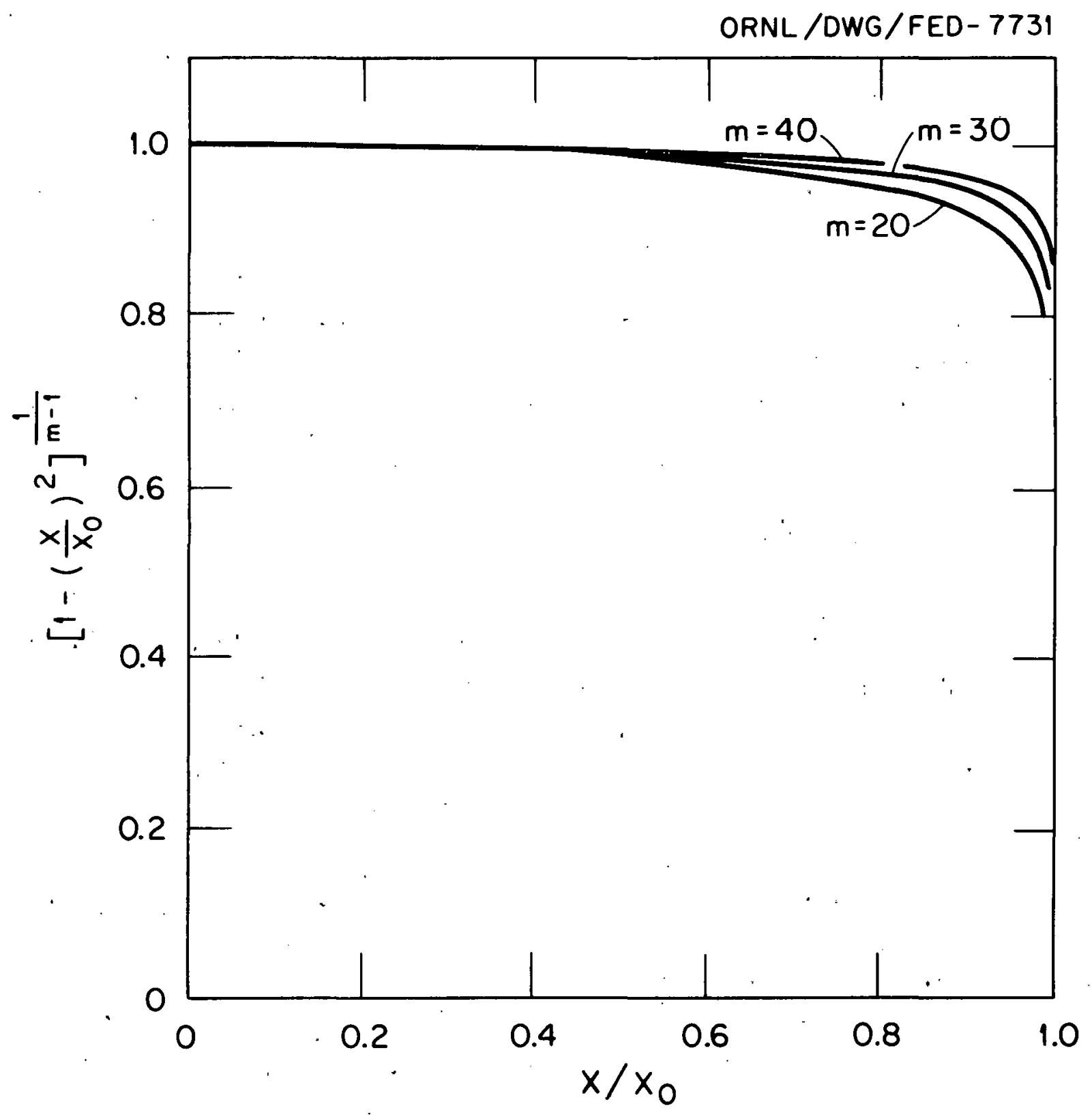

Fig. 4. The shape of the current density distribution for an instantaneous delta-function source. 
$\int_{\substack{\text { half } \\ \text { slab }}} g d y=\left[\frac{m-1}{2 m(m+1)}\right]^{1 /(m-1)} \int_{0}^{\xi_{0}}\left(\xi_{0}^{2}-\xi^{2}\right)^{1 /(m-1)} d \xi$

or

$\bar{g} a=\left[\frac{m-1}{2 m(m+1)}\right]^{1 /(m-1)} \xi_{0}(m+1) /(m-1) \int_{0}^{1}\left(1-z^{2}\right)^{1 /(m-1)} d z$,

where $z=\xi / \xi_{0}$, a is the half-thickness of the slab, and $\bar{g}=\int_{0}^{a} g d y / a$. At the time $t_{0}$ at which the current distributions reach the center line, $\xi_{0}=a \tau_{0}^{-1 /(m+1)}$. Substituting this value in Eq. (18b) and remembering that $t=n \mu_{0} \tau / \rho_{S C}$, we find

$t_{0}=\frac{n u_{0} a^{2}}{\rho_{s c}} \frac{m-1}{2 m(m+1)} \frac{\left[0 \int^{1}\left(1-z^{2}\right)^{1 /(m-1)}\right]}{\bar{g}^{(m-1)}} \cdot$

For values of $\mathrm{m}$ between 20 and 50 , the numerator of the last fraction on the right-hand side of Eq. (19) is within $0.6 \%$ of 0.547 .

In his paper, Wilson considered a cylindrical composite $3.5 \mathrm{~mm}$ in diameter operating at $50 \%$ of critical current. What he meant by the term "critical current" must be inferred from his statement that at $90 \%$ of critical current density the resistivity of the composite is $10^{-14}$ $\Omega-\mathrm{m}$, and at $95 \%$ of critical current density the resistivity of the composite is $10^{-13} \Omega-m$. From these data, we deduce that the critical current density corresponds to a resistivity of the composite of $8.90 \times 10^{-13} \Omega-m$ and $m=43.6$. If we take $\rho_{s c} / n=10^{-7} \Omega-m$, then $g_{c}=J_{c} / J_{0}=\left(8.90 \times 10^{-13} /\right.$ $\left.10^{-7}\right)^{1 / 42.6}=0.761$. At $50 \%$ of critical current, $\bar{g}=0.5 \times 0.761=0.381$. If we take $a=1.75 \mathrm{~mm}$, corresponding to a $3.5-\mathrm{mm}$-thick slab, we find $t_{0}$ $=1.64 \times 10^{11} \mathrm{sec}$ or about 5200 years! Hence, the infinite-medium solution should be applicable for all practical purposes. 
We can find $g(0, t)$ and the corresponding flux-flow resistivity of the composite from

$g(0, t)=\left(\frac{t_{0}}{t}\right)^{1 /(m+1)}\left[\frac{m-1}{2 m(m+1)}\right]^{1 /(m-1)}\left(\frac{n \mu_{0} a^{2}}{\rho_{s c} t_{0}}\right)^{1 /(m-1)}$.

Some results are shown in Table 1.

Table 1. Decay of the current distribution in Wilson's example

\begin{tabular}{ccc}
\hline$t(\mathrm{sec})$ & $g(0, \mathrm{t})$ & $\rho / \mathrm{n}(\Omega-\mathrm{m})$ \\
\hline 1 & 0.689 & $1.32 \times 10^{-14}$ \\
10 & 0.655 & $1.47 \times 10^{-15}$ \\
$10^{2}$ & 0.622 & $1.62 \times 10^{-16}$ \\
$10^{3}$ & 0.591 & $1.80 \times 10^{-17}$ \\
$10^{4}$ & 0.561 & $2.00 \times 10^{-18}$ \\
$10^{5}$ & 0.533 & $2.21 \times 10^{-19}$ \\
$10^{6}$ & 0.506 & $2.46 \times 10^{-20}$ \\
& & \\
\hline
\end{tabular}

These results agree roughly with Wilson's simple estimate of $5 \mathrm{sec}$ to reach $95 \%$ of the critical current density. In fact, the decay is even somewhat faster, reaching about 90\% of critical current density in $1 \mathrm{sec}$. What is most significant is that even though the current density falls slowly with time, the longitudinal flux-flow resistivity $\rho / n$. falls rapidly with time. We may conjecture that if a conductor is charged slowly with time, the longitudinal flux-flow resistivity will approximate the value in the table corresponding to the charging time. If this conjecture is right, the stability of composite conductors against self-field flux jumps should be much improved over what is indicated by Duchateau and Turck. ${ }^{2}$ 
Therefore, in Sect. 4 we turn to the question of what the current distribution is in a magnet. being charged slowly.

\section{LINEAR CHARGING}

The simplest case of gradual charging is the case of linear charging, in which the current in the conductor increases linearly with time. Again, we begin with infinite-medium similarity solutions, but this time we take $g(y, \tau)=\tau^{1 /(m+1)} G\left[y / \tau^{m /(1+m)}\right]$.

Substituting Eq. (21) into Eq. (9), we find that the as yet unknown function $G$ obeys the ordinary differential equation

$(m+1) \frac{d^{2}}{d \zeta^{2}}\left(G^{m}\right)+m \zeta \frac{d G}{d \zeta}-G=0 ; \zeta=y / \tau^{m /(m+1)}$.

Furthermore, the solution in Eq. (21) has the desired property that

$\int_{0}^{\infty} g d y=\tau \int_{0}^{\infty} G(\zeta) d \zeta$,

i.e., that $\int_{0}^{\infty} g d y$ increases linearly with time.

If we integrate. Eq. (22) over $\zeta$, we find, after an integration by parts, that

$$
\left(\frac{d G}{d \zeta}\right)_{\zeta=0}=\frac{-\int_{0}^{\infty} G d \zeta}{m[G(0)]^{m-1}},
$$

which serves as one of the conditions we use to select the solution of Eq. (22) we want. In deriving Eq. (24) we have used another condition, namely,

$G(\infty)=0$.

Equation (22) is not as easy to solve as Eq. (11), and we have integrated 
it numerically. Shown in Fig. 5 are plots of $G$ vs $\zeta$ for $m=20,30$, and 40 , normalized to $G(0)=1$. Values of $G$ for any other normalization of $G$ can be obtained by simultaneously transforming $G$ and $\zeta$ according to

$\left.\begin{array}{l}G^{\prime}=\lambda G \\ \zeta^{\prime}=\lambda^{(m-1) / 2} \zeta\end{array}\right\} 0<\lambda<\infty$,

a transformation that leaves Eqs. (22)-(25) unchanged. Again, notice the nearly rectangular shape of the current distribution.

Let us now continue further analysis of Wilson's problem. . If we define

$t_{1}$ as the total charging time, and define $\bar{g}$ by

$\bar{g} a=\left(\int_{0}^{\infty} g d y\right)_{t=t_{1}}$,

then

$\int_{0}^{\infty} G(\zeta) d \zeta=\frac{\bar{g} a}{\tau_{1}}$.

If $y_{1}$ is the distance the inward diffusing current distribution has penetrated cuwards the center from each edge at time $t_{\eta}$, then

$\zeta_{0}=y_{1} / \tau_{1}^{m /(m+1)}$,

where $\zeta_{0}$ is the value at which $G(\zeta)$ has its first root. Direct numerical calculations show that

$\left.\begin{array}{l}\int_{0}^{w:} G d \zeta=1 \\ \text { and } \\ \zeta_{0}=\frac{m+1}{m}\end{array}\right\}$ when $G(0)=1$, 


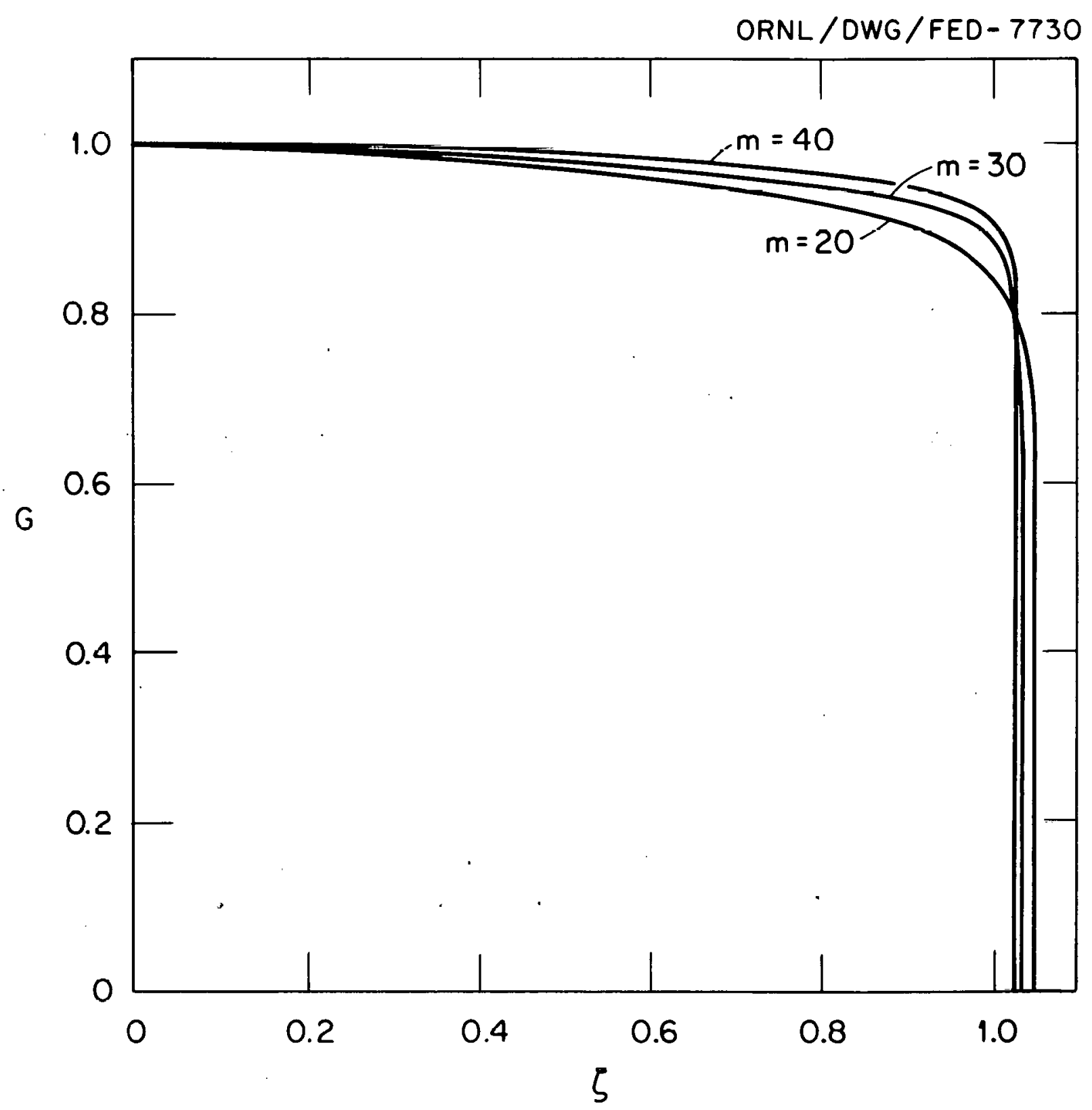

Fig. 5. The shape of the current density distribution for a linearly ramped current source. 
so that

$$
\left.\begin{array}{l}
\int_{0}^{\infty} G d \zeta=\lambda^{(m+1) / 2} \\
\text { and } \\
\zeta_{0}=\left(\frac{m+1}{m}\right) \lambda^{(m-1) / 2}
\end{array}\right\} \text { when } G(0)=\lambda .
$$

Then

$$
\left(\frac{m \zeta_{0}}{m+1}\right)^{2 /(m-1)}=\lambda=\left(\int_{0}^{\infty} G d \zeta\right)^{2 /(m+1)}=\left(\frac{\bar{g} a}{\tau}\right)^{2 /(m+1)},
$$

so that finally

$$
\frac{y_{1}}{a}=\frac{m+1}{m} \tilde{g}^{(m-1) /(m+1)}\left(\frac{\rho_{S c} t_{1}}{n \mu_{0} a^{2}}\right)^{1 /(m+1)} .
$$

Furthermore,

$$
\dot{y}(0, l)=\left(\frac{t}{t_{1}}\right)^{1 /(m+1)} \bar{g}^{2 /(m+1)}\left(\frac{n \mu_{0} a^{2}}{\rho_{s c} t_{1}}\right)^{1 /(m+1)} .
$$

Table 2 shows some values computed using these formulas for Wilson's problem. The first column is the charging time in seconds. We see, as expected, that for long charging times, the longitudinal flux-flow resistivity attained is very smal1. 
Table 2. Linear charging in Wilson's example

\begin{tabular}{cccc}
\hline$t_{1}(\mathrm{sec})$ & $y_{1} / a$ & $g\left(0, t_{1}\right)$ & $\frac{\rho}{n}(\Omega-m)$ \\
\hline 1 & 0.511 & 0.762 & $9.61 \times 10^{-13}$ \\
10 & 0.538 & 0.724 & $1.07 \times 10^{-13}$ \\
$10^{2}$ & 0.567 & 0.688 & $1.18 \times 10^{-14}$ \\
$10^{3}$ & 0.597 & 0.65 .3 & $1.31 \times 10^{-15}$ \\
$10^{4}$ & 0.628 & 0.620 & $1.45 \times 10^{-16}$ \\
$10^{5}$ & 0.662 & 0.589 & $1.61 \times 10^{-17}$ \\
$10^{6}$ & 0.697 & 0.559 & $1.79 \times 10^{-18}$ \\
\hline
\end{tabular}

\section{NEGLECT OF AXIAL DIFFUSION TERM}

Using the solution obtained in the last section, we now study the question of the neglect of the axial diffusion term, i.e., the last term on the right-hand side of Eq. (8). We compare it to the radial diffusion term, which is the first term on the right-hand side of Eq. (8). To facilitate comparison, we first integrate Eq. (8) with respect to y over half the slab. Then we get

$a \frac{\partial}{\partial \tau}\langle g\rangle=\left[\frac{\partial}{\partial y}\left(g^{m}\right)\right]_{y-0}+a \frac{n \rho_{c u}}{\rho_{s i}} \frac{\partial^{2}\langle g\rangle}{\partial x^{2}}$.

We can calculate the first term on the right-hand side using the results of Sect. 4 as follows:

$\left[\frac{\partial}{\partial y}\left(g^{m}\right)\right]_{y=0}=\left[\frac{d}{d \zeta}\left(G^{m}\right)\right]_{\zeta=0}=-\int_{0}^{\infty} G d \zeta=-\frac{\bar{g} a}{\tau_{1}}$.

Then the ratio of the axial to the radial diffusion term is

$\left(\frac{\rho_{c u^{t} 1}}{\mu_{0}}\right) \frac{\partial^{2}}{\partial x^{2}}\left(\frac{\langle g\rangle}{\bar{g}}\right)$. 
Now, because $\langle g\rangle \sim \bar{g}$, it follows that the ratio in Eq. (37) can be on the order of one or larger only over distances on the order of $\left(\rho_{\mathrm{cu}} t_{j} / \mu_{0}\right)^{\frac{3}{2}}$ or less. For $t_{1}=1000 \mathrm{sec}$ and $\rho_{c u}=4 \times 10^{-10} \Omega-m$, this distance is only $0.564 \mathrm{~m}$. Clearly then, axial diffusion is only important near the current leads, and may, in general, be neglected.

\section{SELF-FIELD STABILITY}

The usual method of calculating the self-field stability begins with the assumption of a small rise in the temperature of the conductor. This causes a decrease in the critical current density of the superconductor. In the strict critical-state model, current leaves the outer saturated filaments until the filaments are once again saturated. The current flowing in the copper creates a longitudinal electric field, which, acting upon the current in the filaments, causes dissipation. This dissipation is easily estimated using the "frozen-flux" approximation of Kremlev, Mints, and Rakhmanov. ${ }^{6}$

In the frozen-flux approximation, the current leaving the filaments remains in the copper of the outer sheath of saturated filaments. Hence the average current density there (averaged over a volume containing a few filaments) is constant and equal to $\eta_{c}\left(T_{b}\right)$, where $J_{c}\left(T_{b}\right)$ is the critical current density at the ambient bath temperature $T_{b}$. After a small rise in temperature, $\Delta T$, we have

$n J_{c}\left(T_{b}\right)=n J_{c}\left(T_{b}+\Delta T\right)+E / \rho_{c u}$,

where $E$ is the electric field and the second term on the right-hand side is the current density in the copper. Then

$E=-n \rho_{c u}\left(\frac{d J_{c}}{d T}\right)_{T_{b}} \Delta T=n J_{c}\left(T_{b}\right) \rho_{c u} \frac{\Delta T}{T_{0}}$,

where $T_{0}$ is defined by

$$
T_{0}=-\left(\frac{1}{J_{c}} \frac{d J_{c}}{d T}\right)_{T_{b}} .
$$


Since $E / \rho_{\dot{C} U} \ll J_{C}$ if $\Delta T$ is small, the power density being dissipated in the sheath of saturated filaments is

$Q=E n J_{c}=\left(n J_{c}\right)^{2} \rho_{c u} \frac{\Delta T}{T_{0}}$

When the continuous development of flux-flow resistance is taken into account, there is always a small dissipative power density given by

$\dot{Q}=\left(n J_{c}\right)^{2} \rho_{c}\left(\frac{g(0, t)}{g_{c}}\right)^{m+1}$,

where $J_{c}$ is the same as in Eq. (41), i.e., the current density at which some specified flux-flow resistivity $\rho_{C}$ occurs. If there is a small rise in temperature $\Delta T$, the average current density remains fixed at $\mathrm{J}_{\mathrm{C}}$, but the flux-flow resistivity increases by a factor $\left(1+\Delta T / T_{0}\right)^{m-1}$. [This is because $\mathrm{J}_{0}=\mathrm{J}_{\mathrm{c}} / \mathrm{g}_{\mathrm{c}}$ and $\mathrm{g}_{\mathrm{c}}$ is fixed by the flux-flow resistivity that "defines". the critical current. Hence, $\mathrm{dJ}_{0} / \mathrm{J}_{0} d T=\mathrm{dJ}_{c} / \mathrm{J}_{c} d T=-1 / T_{0}$. If we assume that $J_{c} \sim\left(T_{c r}-T\right) /\left(T_{c r}-T_{b}\right)$, it follows that $T_{0}=T_{c r}$ - $T_{b} \cdot$.] If the increase in the flux-flow resistivity is not sufficient to make it comparable with the resistivity of copper, no current will spill over into the copper.

If we take a charging time of $1000 \mathrm{sec}$ and take $n \mathrm{~J}=5.6 \times 10^{7} \mathrm{~A}-\mathrm{m}^{-2}$ ( $N$ STi at $8 \mathrm{~T}, \mathrm{n} \sim 0,1$ ), we find the steady dissipation $Q$ of $3.02 \mu \mathrm{W}-\mathrm{cm}^{-3}$. If we take $\rho_{\mathrm{Cu}}=4 \times 10^{-10} \Omega-\mathrm{m}$ and $T_{0}=5.6-4.2=1.4 \mathrm{~K}$, then Eq. (41) is less than Eq. (42) only for $\Delta T<3.37 \times 10^{-6} \mathrm{~K}$. The dissipations of Eqs. (41) and (42) are plotted as functions of $\Delta T$ in Fig. 6. (From this example, it is clear that the critical-state model may overestimate by many orders of magnitude the dissipation following a slight temperature rise.) There seems to be no question that the self-field distribution of a slowly charged magnet is much more stable against small temperature perturbations than is indicated by the strict critical-state model.

On the other hand, because of the very rapid rise of $\dot{Q}$ for sufficiently large $\Delta T$ in the flux-flow model, the conductor cannot be stable against arbitrarily large temperature rises. To estimate how large a temperature rise we can stand, we have plotted the cooling curve $h P \Delta T / A=2 h \Delta T / R$ for a heat transfer coefficient of $h=0.1 \mathrm{~W}-\mathrm{cm}^{-2}$. Here $P$ is the perimeter 


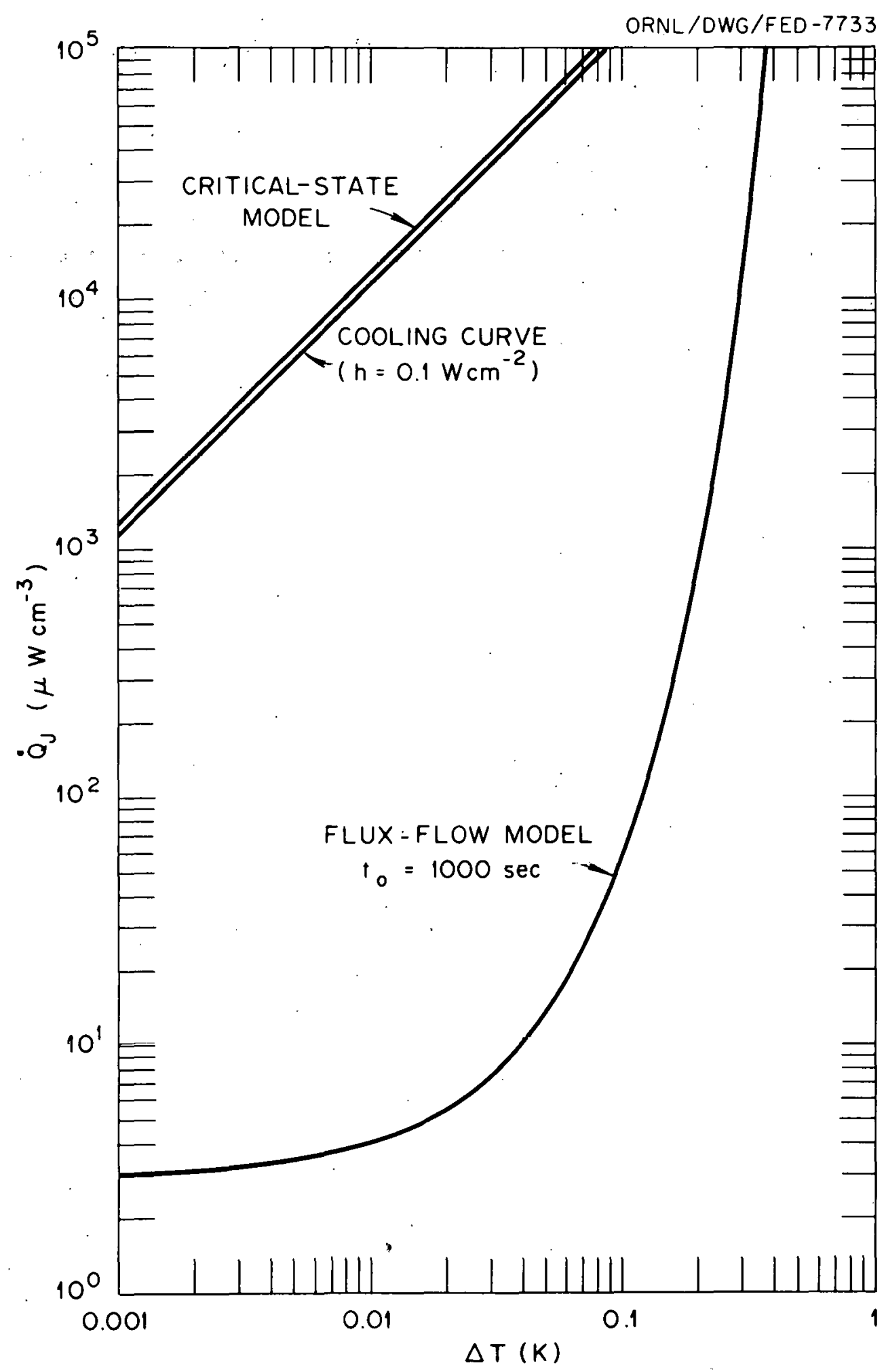

Fig. 6. The dissipation as a function of temperature rise according to the critical-state and flux-flow models. 
of the conductor, $A$ is its cross-sectional area, and $R$ is its radius. The cooling curve and the heat production curve of the flux-flow model intersect at temperature increases of $2.64 \times 10^{-6} \mathrm{~K}$ and $0.458 \mathrm{~K}$. The former temperature increase gives the order of magnitude of the temperature excess of the conductor over the bath temperature due to the steady production of $3.02 \mu \mathrm{W}-\mathrm{cm}^{-3}$. The latter temperature gives the temperature excess at which heating first begins to exceed cooling. The conductor in this example is almost surely stable against perturbations on the order of $0.1 \mathrm{~K}$ when $t_{1}=1000 \mathrm{sec}$. 


\section{REFERENCES}

1. M. N. Wilson, Proc. 5th Int7. Conf. on Magnet Technology, pp. 21-25 (1975); also published as RL-75-060, Rutherford Laboratory, Chilton, Oxfordshire, England (1975).

2. J. J. Duchateau and B. Turck, J. App 1. Phys. 46, 4989 (1975).

3. M. N. Wilson, Report SMR-1, Rutherford Laboratory, Chilton, Oxfordshire, England (1977):

4. G. Birkhoff, Hydrodynamics, 2nd ed., Princeton University Press, Princeton, New Jersey, 1960.

5. W. F. Ames, Ind. Eng. Chem. Fundamentals 4, 72 (1965).

6. M. G. Kremlev, R. G. Mints, and A. L. Rakhmanov, Dok1. Akad. Nauk SSR [Sov. Phys. Doklady] 21 (5), 270 (1976). 
THIS PAGE

\section{WAS INTENTIONALLY LEFT BLANK}


ORNL/TM-5986

INTERNAL DISTRIBUTION

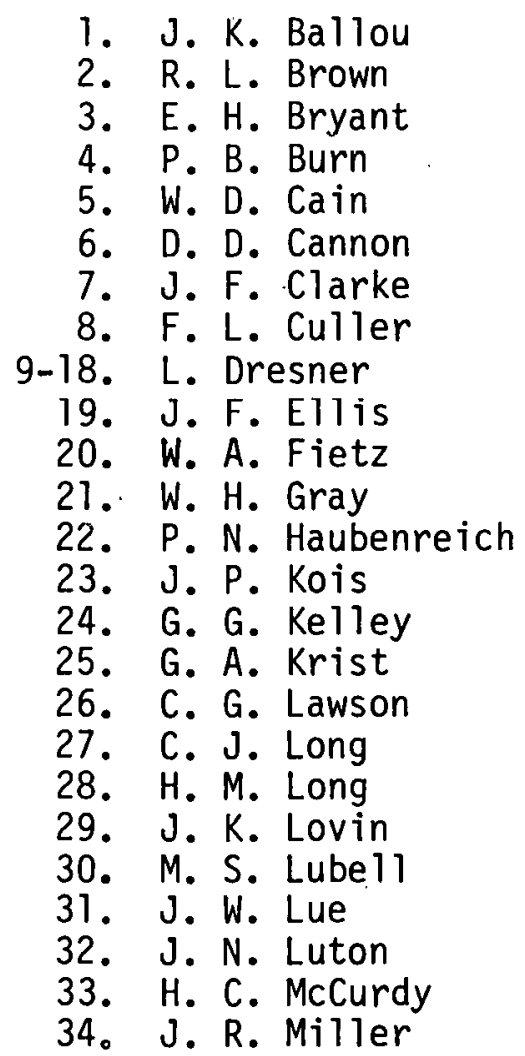

35. R. V. Miskell

36. 0. B. Morgan

37. L. W. NeIms

38. H. Pih

39. H. Postma

40. M. Roberts

41. M. W. Rosenthal

42. R. E. Schwall

43. T. E. Shannon

44. S. S. Shen

45. J. E. Simpkins

46. D. Steiner

47. W. C. T. Stoddart

48. P. B. Thompson

49. P. L. Walstrom

50. H. T. Yeh

51. A. Zucker

52-53. Central Research Library

54. Fusion Energy Division Reports Office

55. Document Reference Section

56-57. Laboratory Records Department

58. Laboratory Records - ORNL RC

59. ORNL Patent Office

60-61. Fusion Energy Division Library

\section{EXTERNAL DISTRIBUTION}

62. E. Adam, Airco, 100 Mountain Avenue, Murray Hil1, NJ 07974

63. V. D. Arp, National Bureau of Standards, Boulder, CO 80302

64. J. W. Beal, Division of Magnetic Fusion Energy, ERDA, Mail Stop G-234, Washington, DC 20545

65. R. W. Boom, 513 Engineering Research Building, University of Wisconsin, Madison, WI 53706

66. A. Clark, National Bureau of Standards, Boulder, CO 80302

67. D. L. Coffey, American Magnetics Inc., P.0. Box R, Oak Ridge, TN 37830

68. W. Coles, NASA Louis Research Laboratory, Cleveland, $\mathrm{OH} 44108$

69. D. N. Cornish, Lawrence Livermore Laboratory, P.0. Box 808, Livermore, CA 94550

70. M. D'Agostino, Grumman Aerospace Corporation, Bethpage, NY 11714

71. R. W. Derby, Magnetics Engineering Associates, 247 Third Street, Cambridge,' $M A$ O 02141

72. D. A. Dingee, Manager, Fusion Programs, Battelle-Northwest, Battelle Boulevard, Richland, WA 99352 
73. Director, Research and Technical Support Division, ERDA, Oak Ridge Operations, Oak Ridge, TN 37830

74. R. W. Fast, Manager, Experimental Facilities, National Accelerator Laboratory, P.0. Box 500, Batavia, IL 60510

75. J. J. Ferrante, General Electric Co., Schenectady, NY 12345

76. F. Fickett, National Bureau of Standards, Boulder, CO 80302

77. J. File, Princeton University, Plasma Physics Laboratory, Princeton, NJ 08540

78. H. K. Forsen, Laser Enrichment Department, Exxon Nuclear Co., Inc., 777-106th Avenue, NE, C-00777, Bellevue, WA 98009

79. H. P. Furth, Princeton University, Plasma Physics Laboratory, Princeton, NJ 08540

80. W. S. Gilbert, Lawrence Berkeley Laboratory, University of California, Berkeley, CA 94120

81. R. W. Gould, Department of Applied Physics, California Institute of Technology, Pasadena, CA 91109

82. E. Gregory, Airco, 100 Mountain Avenue, Murray Hil1, NJ 07974

83. D. S. Hackley, General Dynamics, Convair Division, P.0. Box 80847, San Diego, CA 92138

84. W. V. Hassenzahl, Los Alamos Scientific Laboratory, P.0. Box 1663, Los Alamos, NM 87544

85. C. D. Henning, Division of Magnetic Fusion Energy, ERDA, Mai1 Stop G-234, Washington, DC 20545

86. G. K. Hess, Division of Magnetic Fusion Energy, ERDA, Mail Stop G-234, Washington, DC 20545

87. R. L. Hirsch, Deputy Manager Science and Technology Division, Exxon Corp., 1251 Avenue of the Americas, New York, NY 10020

88. M. 0. Hoenig, Massachusetts Institute of Technology, National Magnet Laboratory, 170 Albany Street, Cambridge, MA 02139

89. C. K. Jones, Manager, Cryogenic Research Laboratory, Westinghouse Electric Corporation, Research and Development Center, Pittsburgh, PA 15235

90. E. E. Kintner, Division of Magnetic Fusion Energy, ERDA, Mai1 Stop G-234, Washington, DC 20545

91. H. L. Laquer, Los Alamos Scientific Laboratory, P.0. Box 1663, Los Alamos, NM 87544

92. C. Laverick, 543 Hampshire Lane, Boling Brook, IL 60439

93. D. B. Montgomery, Massachusetts Institute of Technology, National Magnet Laboratory, 170 Albany Street, Cambridge, MA 02139

94. F. Moon, Department of Theoretical and Applied Mechanics, Cornell University, Ithaca, NY 14850

95. C. Oberly, Aero Propulsion Laboratory, Power Distribution Branch, Wright-Patterson Air Force Base, $\mathrm{OH}, 45433$

96. J. R. Powe11, Brookhaven National Laboratory, Upton, NY 11973

97. J. R. Purcel1, General Atomic Company, P.0. Box 81608, San Diego, CA 92138

98. P. Reardon, Princeton Plasma Physics Laboratory, Princeton University, P.0. Box 451, Princeton, NJ 08540

99. R. P. Reed, National Bureau of Standards, Boulder, C0 80302

100. J. D. Rogers, Q-26, Los Alamos Scientific Laboratory, P.0. Box 1663, Los Alamos, NM 87544 
101. D. J. Rose, Department of Nuclear Engineering, Massachusetts Institute of Technology, Cambridge, MA 02139

102. C. H. Rosner, Intermagnetics General Corporation, Charles Industrial Park, New Karner Road, Guilderland, NY 12084

103. W. B. Sampson, Brookhaven National Laboratory, Upton, Long Island, NY 11973

104. S. St. Lorant, Stanford Linear Accelerator Center, Sand Hill Road, Palo Alto, CA 94304

105. Z. J. J. Stekly, Magnetic Corporation of America, 179 Bear Hill Road, Waltham, MA 02154

106. L. D. Stewart, Princeton Plasma Physics Laboratory, Princeton University, P.0. Box 451, Princeton, NJ 08540

107. B. P. Strauss, Fermi National Accelerator Laboratory, P.0. Box 500, Batavia, IL 60510

108. C. E. Taylor, Lawrence Livermore Laboratory, P.0. Box 808, L-384, Livermore, CA 94551

109. R. Thomas, General Atomic Company, P.0. Box 81608, San Diego, CA 92138

110. C. von Keszycki, Grumman Aerospace Corporation, Research Center, Building 26, Bethpage, NY 11714

111. S. T. Wang, Argonne National Laboratory, 9700 South Cass Avenue, Argonne, IL 60439

112. J. P. Waszczak, Manager, Energy Programs, General Dynamics, Convair Division, P.0. Box 80847, San Diego, CA 92138

113. J. M. Williams, Division of Magnetic Fusion Energy, ERDA, Mail Stop G-234, Washington, DC 20545

114. S. L. Wipf, Q-26, Los Alamos Scientific Research Laboratory, P.0. Box 1663, Los Alamos, NM 87544

115. W. W. Withee, Director, Energy Systems, General Dynamics, Convair Division, P.O. Box 80847, San Diego, CA 92138

116. J. Wong, Supercon, Incorporated, 9 Erie Drive, Natick, MA 01760

117. H. H. Woodson, Chairman, Department of Electrical Engineering, University of Texas, Austin, TX 78712

118. J. L. Young, Westinghouse Electric Corporation, P.0. Box 10864, Pittsburgh, PA 15236

119. E. J. Zlurys, Division of Magnetic Fusion Energy, ERDA, Mail Stop G-234, Washington, DC 20545

120. Dr. Y. Aiyama, Electrotechnical Laboratory, 5-4-1 Mukodaj-cho, Tanashi-City, Tokyo, Japan

121.: Dr. Gunther Bogner, Forschungslaboratorien, Siemens Aktienge Sel1shaft, Postfach 325, D8520 Erlanger 2, West Germany

122. Dr. Gaston Bronca, Department du Synchrotron Saturne, CEN/Saclay, F91190, Gif-sur-Yvette, France

123. Dr. Henri Desportes, STIPE, CEN/Saclay, F91190, Gif-sur-Yvette, rrance

124. Professor G. W. Donaldson, School of Electrical Engineering, University of New South Wales, P.0. Box 1, Kensington, New South Wales 2033, Australia

125. Dr. Yoshio Furuto, Chief of Superconducting Group, Central Research Laboratory, Furukawa Electric Company, Ltd., 9-15, 2-Chome, Futaba, Shinagawa-ku, Tokyo 141, Japan 
126. Prof. Dr. W. F. Gauster, c/o, o. Professor Dr. techn. H. Kirchmayr, Director, Institut für Experimentalphysik, der Technischen Hochschule in Wien, A-1040 Vienna, Karlsplatz 13, Austria

127. Dr. Roger Hancox, UKAEA, Culham Laboratory, Abingdon, OXON, Ox14-3DB, England

128. Professor W. Heinz, Gesellschaft für Kernforschung, Institut für Experimental Kernphysik, 75 Karlsruhe, Postfach 3640, West Germany

129. Toru Hiraoka, Japan Atomic Energy Research Institute, Tokai Research Establishment, Tokai-Mura, Naka-Gun, Ibaraki-Ken, Japan

130. Dr. Seijiro Ihara, Energy Division, Electrotechnical Laboratory, 5-4-1 Mukodai-cho, Tanashi-City, Tokyo, Japan

131. Or. Masatami Iwamoto, Central Research Laburatory, Mitsubishi Electric Corporation, 80 Nakano, Minamishimizu, Amagasaki, Hyogo Prefecture, Japan 660

132. Dr. T. Kasahara, Hitachi Works, 3-1-1 Saiwai-cho, Hitachi-shi, Ibaraki-ken 317, Japan

133. Dr. Alfred A. Koch, Brown, Boveri and Company, Itenhard 29, CH-5620 Bremgarten, $\mathrm{CH}-8050$ Zurich, Bade, Switzerland

134. Dr. Albert Knobloch, Max-Planck Institut für Plasmaphysik, Abteilung Technik, 8046 Garching bei Munich, West Germany

135. Dr. Peter Komarek, Institut für Experimental Kernphysik, 75 Karlsruhe, Postfach 3640, West Germany

136. Dr. Kenichi Koyama, Electrotechnical Laboratory, 5-4-1 Mukodai-cho, Tanashi-City, Tokyo, Japan

137. Dr. Kunishige Kuroda, Hitachi, Ltd., Central Research Laboratory, 1-280, Higa Shiko Igakubo, Kokubumj $i$, Tokyo 185, Japan

138. Dr. Alberto Martine11i, DSS SEDAP, B.P. No. 2, CEN/Saclay, F91190, Gif-sur-Yvette, France

139. Max-Planck Institut für Plasmaphysik, 8046 Garching bei München, Federal Republic of Germany

140. Professor T. Ogasawara, Department of Physics, Coīiege of Science and Engineering, Nihon University, Kanada-Surugadai, Chiyoda-ku, Tok.yo, Japan

141. Dr. H. Ogiwara, Toshiba Research and Development Center, I Komukai Tsohibacho, Saiwai-ku, Kawasaki-City, Kanagawa 210, Japan

142. Dr. Jacques Parain, CEN/Saclay, B.P. No. 2, F91190 Gif-sur-Yvette, France

143. Dr. G. Sacerdoti, Laboratoroi Nazionali, Cas. Postale 70, 00044 Frascati, Italy

144. Dr. Yoshio Sawada, Manager of Advanced Engineering Group, Heavy Apparatus Engineering Laboratory, Tokyo Shibaura Electric Co., Ltd. 4, 2-Chome, Suehiro-cho, Tsurumi-ku, Yokohama, Japan

145. Director K. Schmitter, Max-Planck Institut fü Plasmaphysik, 8046 Garching bei Munich, West Germany

146. Dr. S. Shimamoto, Japan Atomic Energy Research Institute, Tokai Research Establ ishment, Toka i-mura, Naka-gun, Ibaraki-ken, Japan

147. Dr. M. Spadoni, Laboratori Nazionali, Cas. Postale 70, 00044 Frascati, Italy

148. Dr. Kyoji Tachikawa, Chief of Electric Materials Laboratory, National Research Institute for Metals, 3-12, 2-Chome, Nakameguro, Meguru-ku, Tokyo, Japan 
149. Professor D. T. Uchida, Nuclear Engineering Department, University of Tokyo, Tokyo, Japan

150. Dr. Col in Walters, Technology Division, Building R25, Rutherford Laboratory, Chilton, Didcot, Oxfordshire, OX1100X, England

151. Dr. M. Yamamoto, Toshiba, Tsurumi Works, 4, Suehiro-choizchome, Tsurumi-ku, Yokohama, 230, Japan

152. Professor Ko Yasukochi, Dept. of Physics, College of Science and Engineering, Nihon University, Kanada-Surugadai, Chiyoda-ku, Tokyo, Japan

153-179. Technical Information Center, P.0. Box 62, Oak Ridge, TN 37830 